\title{
Stress intensity factors for cracked cold-drawn steel wires under tensile loading
}

\author{
B. $\operatorname{Lin}^{1} \& \mathrm{G} \cdot \mathrm{Lu}^{2}$

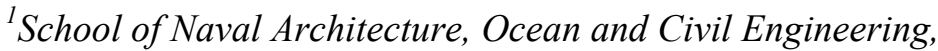 \\ Shanghai Jiaotong University, People's Republic of China \\ ${ }^{2}$ Department of Bridge Engineering, \\ Tongji University, People's Republic of China
}

\begin{abstract}
Cold-drawn steel wires have the excellent mechanical properties of strength and toughness and are widely used in prestressed concrete structures. During cold-drawn operations residual stresses are generated in steel wires. In this paper, by the residual stress distribution from the finite element analysis (FEA), a weight function method (WFM) has been used to evaluate the effective stress intensity factors (SIFs) for cracked cold-drawn steel wires under tensile loading. The calculation results have been compared with those obtained by the two-dimensional (2D) FEA, which considers the residual stress redistribution in the presence of a crack. In the present study, the effective SIFs calculated using the WFM have shown a good agreement with those derived from the 2D FEA. Keywords: stress intensity factor, residual stress, weight function, cold-drawn steel wire, finite element analysis.
\end{abstract}

\section{Introduction}

In the cold-drawn procedure, the tensile strength and toughness of steel wires increase, but a residual stress field also appears in them. After the treatment of a further drawing with a very small area reduction or a combination of heating and stretching the wire, the decreased residual tensile stresses still exist in the steel wire surface [1]. It is well known that the presence of residual tensile stresses will reduce the fatigue life of steel wires. With increasing in residual tensile stresses, the fatigue crack growth rate increases for stress ranges close to the fatigue limit [2]. The calculation of the SIF is one of key factors in fatigue life 
determination of the components. The superposition principle, which assumes that the stresses applied by external loading superimpose linearly on residual stresses, can be used to calculate the effective SIFs in a cracked body in which a residual stress field acts [3]. If the weight function, which depends only on the geometry of the body, is obtained, the contribution of residual stresses on the effective SIFs for cracks in mode I can be determined by the WFM [4]. As for cold-drawn steel wires under tensile loading, the net stresses in the steel wires under service loading remain tensile. When a crack is produced such that it lies wholly in a tensile region the faces of the crack will remain fully open and the applicability of the linear superposition principle is valid [5]. Additionally, the produced crack will bring the residual stresses ahead of a crack tip to redistribute, $[5,6]$. So, the availability of the WFM, which assumes that the residual stress distribution keeps invariable, must be validated when dealing with residual stresses and fatigue crack growth.

In this paper, the 2D FEA, which considers the residual stress redistribution in the presence of a crack, is carried out to validate the WFM for calculations of effective SIF for cracked cold-drawn steel wires under tensile loading. Section 2 presents the 2D model for the cracked steel wire. Calculations of the effective SIF by the weight function analysis are described in Section 3 and by the 2D FEA in Section 4. Section 5 gives the corresponding analysis results and discussion. The present work is concluded in Section 6.

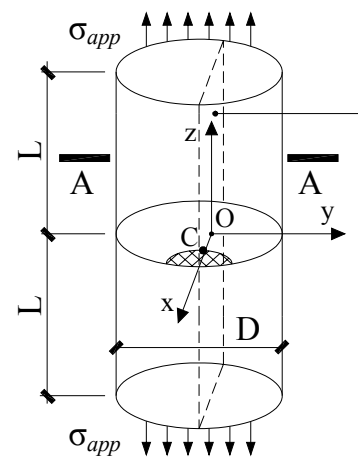

(a)

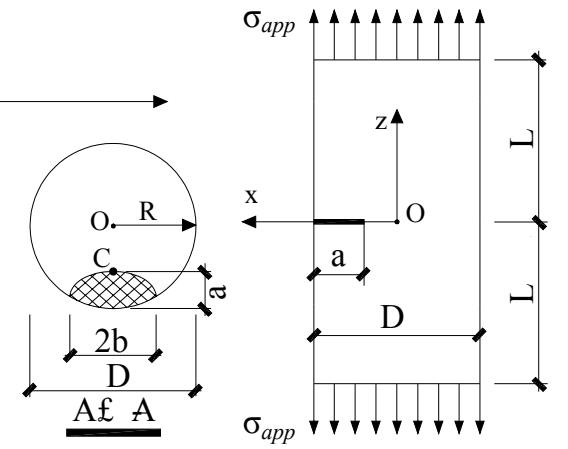

(b)

(c)

Figure 1: $\quad 3 \mathrm{D}$ geometry and 2D model of a wire used in the analysis.

\section{Two-dimensional model}

The surface crack of the cold-drawn steel wires produced by mechanical fatigue can be assumed to be a semi-elliptical geometry $(a / b<1$, a is the crack depth or minor axis of the ellipse, $b$ the major axis of the ellipse), perpendicular to the tensile loading direction [7], as show in fig. 1(a), 1(b), and the maximum SIF value was obtained at the crack center $\mathrm{C}$, according to the results given by Astiz [8]. Following a well known purely linear elastic solution in the vicinity of 
a 3D crack with an arbitrary curved front [9], the calculation of the SIF can be based on 2D plane strain hypothesis on the longitudinal section of a cracked cold-drawn steel wire under tensile loading, fig. 1(c).

\section{Weight function analysis}

As for a cracked cold-drawn steel wire under tensile loading, the effective SIF at the crack front is decided by the combined effect of local residual stresses and the stresses applied by external loading. According to the linear superposition principle, the effective SIF $\left(K_{\text {eff }}\right)$ is the algebraic sum of the external SIF $\left(K_{a p p}\right)$ due to the external applied loads and the residual SIF $\left(K_{\text {res }}\right)$ due to the residual stresses, and can be expressed as:

$$
K_{\text {eff }}=K_{\text {app }}+K_{\text {res }}
$$

\subsection{Residual SIF}

It is well known that, once the weight function is determined for a particular cracked body, the SIFs can be obtained for any stress field by an appropriate integration. So, in this section, the WFM is used to evaluate the residual SIF $\left(K_{\text {res }}\right)$.

Based on the FEA [1], the distribution of the residual stresses for colddrawn steel wires with a thermomechanical treatment is shown in fig. 2, where $r$ is the polar coordinate of the wire and $\mathrm{R}$ the radius of the wire, and the equation used for the calculation of the residual stresses in the plane problem is as follows:

$$
\begin{aligned}
\sigma_{r e s}(r / R)= & -235.71+411.88 \cdot r / R-987.84 \cdot(r / R)^{2}+3133.93 \cdot(r / R)^{3} \\
& -3257.62 \cdot(r / R)^{4}+1029.93 \cdot(r / R)^{5} .
\end{aligned}
$$

In the edge-cracked 2D model, fig. 1c, a residual SIF can be calculated by integrating the weight function $m(x, a)$ and the residual stress distribution $\sigma_{\text {res }}(x)$ acting on the crack length a [10], which is expressed as follows:

$$
K_{\text {res }}=\int_{0}^{a} \sigma_{r e s}(x) m(x, a) d x
$$

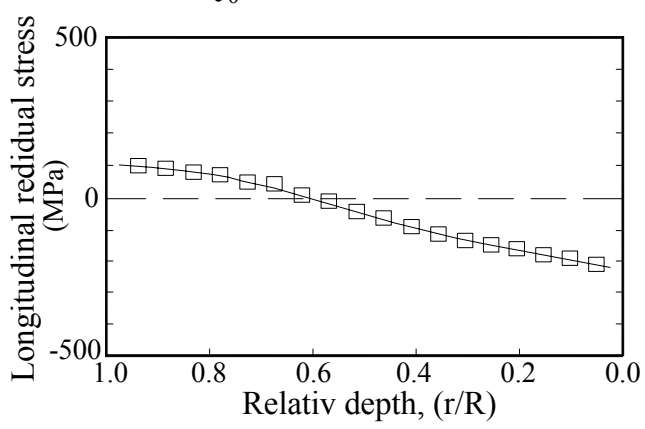

Figure 2: $\quad$ Residual stress distribution for a steel wire used in the analysis. 
The analytical weight function for an edge crack in a finite width plate, as shown in fig. 3, was given by Kaya and Erdogan [11]:

$$
m(x, a)=\frac{2}{\sqrt{\pi a}} \frac{G\left(\frac{x}{a}, \frac{a}{D}\right)}{\left(1-\frac{a}{D}\right)^{3 / 2} \sqrt{1-\left(\frac{x}{a}\right)^{2}}}
$$

where

$$
\begin{aligned}
& G\left(\frac{x}{a}, \frac{a}{D}\right)=g_{1}\left(\frac{a}{D}\right)+g_{2}\left(\frac{a}{D}\right) \cdot \frac{x}{a}+g_{3}\left(\frac{a}{D}\right) \cdot\left(\frac{x}{a}\right)^{2}+g_{4}\left(\frac{a}{D}\right)\left(\frac{x}{a}\right)^{3}, \\
& g_{1}\left(\frac{a}{D}\right)=0.46+3.06 \cdot \frac{a}{D}+0.84\left(1-\frac{a}{D}\right)^{5}+0.66\left(\frac{a}{D}\right)^{2}\left(1-\frac{a}{D}\right)^{2}, \\
& g_{3}\left(\frac{a}{D}\right)=6.17-28.22 \frac{a}{D}+34.54\left(\frac{a}{D}\right)^{2}-14.39\left(\frac{a}{D}\right)^{3}-\left(1-\frac{a}{D}\right)^{3 / 2} \\
& \quad-5.88\left(1-\frac{a}{D}\right)^{5}-2.64\left(\frac{a}{D}\right)^{2}\left(1-\frac{a}{D}\right)^{2}, \\
& +2\left(\frac{a}{D}\right)=-6.63+25.16 \frac{a}{D}-31.04\left(\frac{a}{D}\right)^{2}+14.41\left(\frac{a}{D}\right)^{3} \\
& +5)^{3 / 2} \\
& +5.04\left(1-\frac{a}{D}\right)^{5}+1.98\left(\frac{a}{D}\right)^{2}\left(1-\frac{a}{D}\right)^{2} .
\end{aligned}
$$

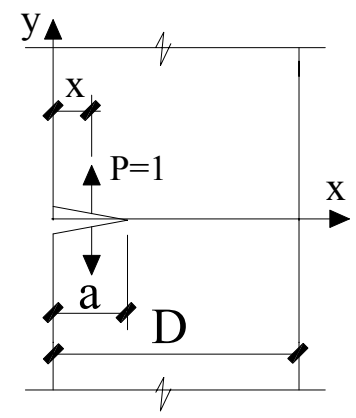

Figure 3: Weight function notation for an edge crack in a finite width plate. 


\subsection{External SIF}

An empirical solution of the SIF for a single edge cracked plate applied under the axial stress, fig. 1(c), given by Tada et al. [12] is used to calculate the external SIF $\left(K_{a p p}\right)$, which is expressed as follows:

$$
K_{a p p}=\sigma_{a p p} \sqrt{\pi a} \cdot F(a / D)
$$

where $\sigma_{a p p}$ is the external applied stress, D the diameter of the steel wire and $F$ $(a / D)$ given by:

$$
F(a / D)=\sqrt{\frac{2 D}{\pi a} \tan \frac{\pi a}{2 D}} \cdot \frac{0.752+2.02 \frac{a}{D}+0.37\left(1-\sin \frac{\pi a}{2 D}\right)^{3}}{\cos \frac{\pi a}{2 D}}
$$

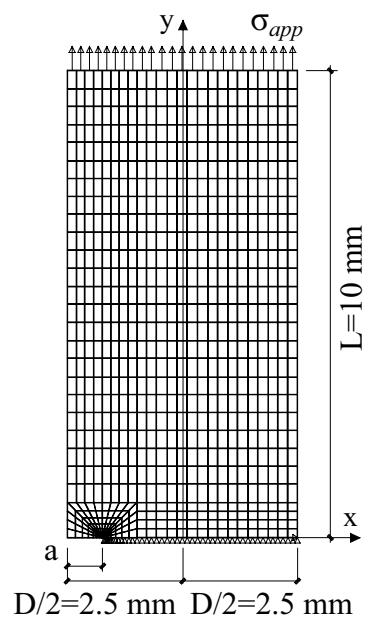

Figure 4: $\quad 2 D$ finite element model.

\section{Finite element analysis}

\subsection{D finite element model}

2D finite element model of the cracked steel wire was developed for numerical stress analysis. A detail of a typical mesh was shown in fig. 4. A different mesh was used for each crack length to present the equivalent level of mesh refinement. The ABAQUS finite element program [13] was chosen for the present analysis. 8-node biquadratic solid element for plane strain available in the ABAQUS code element library was used in the model, and the quarter point technique, used for numerical evaluation of the SIF, was applied to improve precision of the results. Due to symmetry, as shown in fig. 1(c), only the top half-plane was modelled for the specimen with a homogeneous linear elastic 
material using a Young's modulus, $E$, of $207 \mathrm{GPa}$, Poisson's ratio, $v$, of 0.3 and Limit strength, $f_{\text {ptk }}$, of $1940 \mathrm{MPa}$.

The SIF for the elastic singular stress field was calculated by means of the $J$-integral for 10 different paths, which was obtained using the domain integral formulation in ABAQUS. Calculations coupled with the residual stress field were carried out for each crack length. Two levels of applied loading, $\sigma_{a p p}=0.4$ and $0.7 f_{\text {ptk }}$, were employed and applied to the top edge of the model. From the average $J$-integral value, the effective SIF was computed.

\subsection{Verification of the finite element model}

In order to verify the finite element model's accuracy to calculate SIFs for different crack lengths, comparisons between these solutions and empirical solutions given by eqn. (5) were made only under tension loading. The normalised SIF, $K_{I} / \sigma_{a p p} \sqrt{\pi a}$, is presented in fig. 5, showing a fairly good agreement and all differences being within $1 \%$. Based on these results, the present finite element model was considered suitable for the 2D analysis of cracked cold-drawn steel wires under tensile loading.

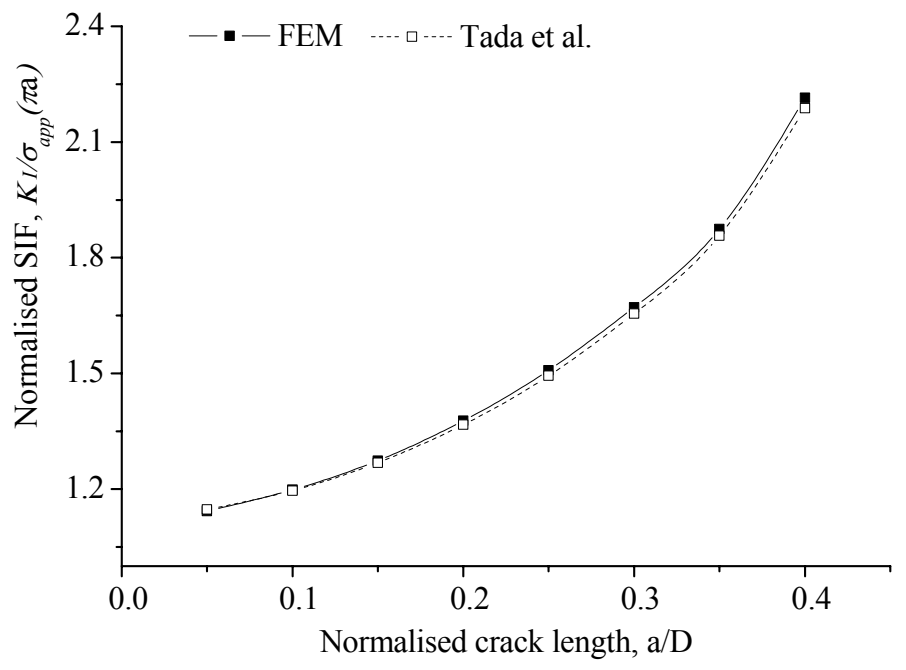

Figure 5: Comparison of $K_{I} / \sigma_{a p p} \sqrt{\pi a}$ between FEM and Tada et al.'s calculations.

\subsection{Residual stresses}

The initial stress method illustrated by Lei et al. [14] can be used to introduce a residual stress distribution into the finite element model as an initial condition, and a fine mesh has been employed to provide for an accurate description of the residual stress distribution in the model. But the introduced residual stresses are 
not self-equilibrating; usually resulting in a stress distribution that differs significantly from desired one. This problem was overcome by using two load steps in the linear elastic analysis. In the first step, reactions required to balance the out-of-balance load vector caused by the residual stresses were derived with all degrees of freedom constrained and no other applied loadings. In the second step, the obtained reactions were converted to applied force, and an equilibrium step analysis was executed for the uncracked model with the standard boundary conditions. After this procedure, the contour of the residual stresses (unit: $\mathrm{MPa}$ ) is presented in fig. 6. It is shown that the residual stress distribution in the uncracked model accords well with the target distribution shown in fig. 2.
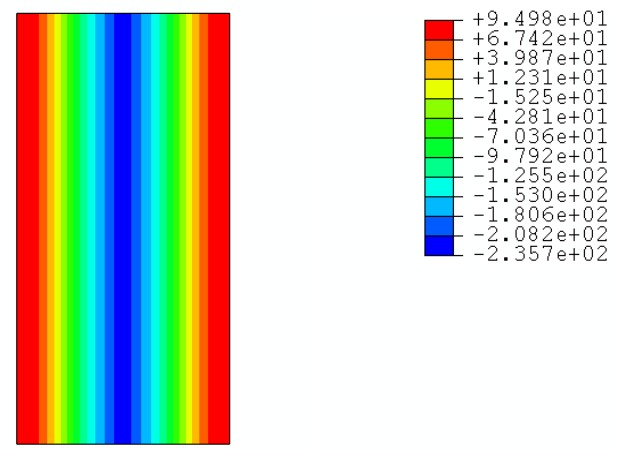

Figure 6: Contour of residual stresses in the uncracked model.

\subsection{Residual stress redistribution}

After the residual stresses are introduced into the uncracked model, a crack can be introduced into the FE model by releasing nodes located on the crack face until the desired crack length is reached. When a node is released from its displacement constraint, the reactions previously acting on the node disappear, and moreover, the equilibrium state in the elements around the node is also changed. Subsequently, a new equilibrium state is re-established. The shift of the equilibrium state will bring the residual stresses in these elements to redistribute.

\section{Analysis results and discussion}

Both the WFM and the FEM described above were used to evaluate the variation of the effective SIF as a function of the crack length. The effective SIFs calculated from the WFM and those derived from the FEA are compared in fig. 7 for seven different crack lengths. From this figure, one can find that, for different crack lengths under two loading levels, the maximum difference in values between the two methods is $0.87 \%$, which exhibits a good mutual agreement. Comparison results between the FEA and the WFM showed that, when calculating effective SIFs with a linear elastic analysis for cracked cold-drawn 
steel wires under tensile loading, the phenomenon of redistribution was not obvious to residual stress problems, which keeps a good agreement with the results for cold-worked cracked holes given by Moreira et al. [15].

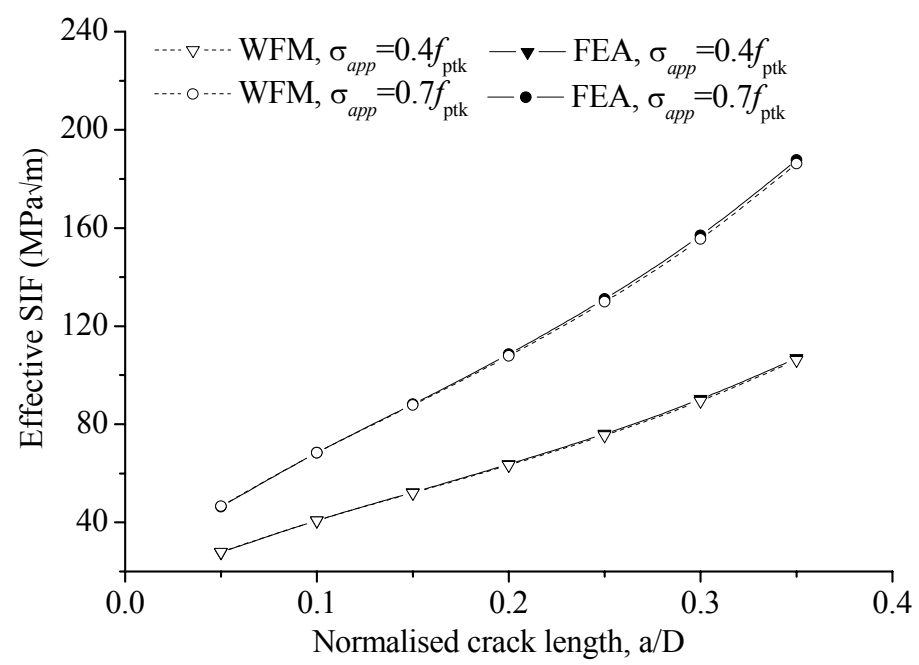

Figure 7: Comparison of residual SIF between weight function and finite element results.

\section{Conclusions}

In this paper, we have demonstrated that, according to the obtained residual stress fields from documents, the effective SIF can be calculated for a cracked body using either the WFM or the FEA. The following conclusion can be drawn: the reliability of the WFM results is demonstrated by comparing with FE calculations, and the complexity of the analysis is remarkably reduced.

\section{References}

[1] Elices, M., Influence of residual stresses in the performance of cold-drawn pearlitic wires. Journal of Materials Science, 39(12), pp. 3889-3899, 2004.

[2] Llorca, J. \& Sanchez-Galvez, V., Fatigue limit and fatigue life prediction in high strength cold drawn eutectoid steel wires. Fatigue and Fracture of Engineering Materials \& Structures, 12(1), pp. 31-45, 1989.

[3] Parker, A.P., Stress intensity factors, crack profiles and fatigue crack growth rates in residual stress fields. ASTM STP 776, pp. 13-31, 1981.

[4] Glinka, G. \& Shen, G., Universal features of weight functions for cracks in mode I. Engineering Fracture Mechanics, 40(6), pp. 1135-1146, 1991. 
[5] Wilks, M.D.B., Nowell, D. \& Hills, D.A., The evaluation of stress intensity factors for plane cracks in residual stress fields. Journal of Strain Analysis for Engineering Design, 28(3), pp. 145-152, 1993.

[6] Lee, Y.B., Chung, C.S., Park, Y.K. \& Kim, H.K., Effects of redistributing residual stress on the fatigue behavior of SS330 weldment. International Journal of Fatigue, 20(8), pp. 565-573, 1998.

[7] Toribio, J. \& Toledano, M., A fracture criterion for prestressing steel cracked wires. Proc. Of the 2nd Int. Conf. On Advances in Steel Structures, eds. S.L. Chan \& J.G. Teng, Elsevier: New York, pp. 947-954, 1999.

[8] Astiz, M.A., An incompatible singular elastic element for two- and threedimensional crack problems. International Journal of Fracture, 31(2), pp. 105-124, 1986.

[9] Hartranft, R.J. \& Sih, G.C., Stress singularity for a crack with an arbitrarily curved front. Engineering fracture Mechanics, 9(3), pp. 705718, 1977.

[10] Cartwright, D.J., Stress intensity factor determination (Chapter 2). Developments in Fracture Mechanics, eds. G.G. Chell, Applied Science: London, pp. 29-66, 1980.

[11] Kaya, A.C. \& Erdogan, F., Stress intensity factors and COD in an orthotropic strip. International Journal of Fracture, 16(2), pp. 171-190, 1980.

[12] Tada, H., Paris, P.C. \& Irwin, G.R., (eds). The Stress Analysis of Cracks Handbook, Del Research Corporation: Hellertown, Pennsylvania, 1973.

[13] Hibbitt, B., Karlsson, B. \& Sorensen, P., (eds). ABAQUS/Standard User's Manual, version 6.1, Hibbitt, Karlsson \& Sorensen Inc.: Rode Island, RI, 2001.

[14] Lei, Y., O'Dowd, N.P. \& Webster, G.A., Fracture mechanics analysis of a crack in a residual stress field. International Journal of Fracture, 106(3), pp. 195-216, 2000.

[15] Moreira, P.M.G.P., De Matos, P.F.P. \& Pinho, S.T., The residual stress intensity factors for cold-worked cracked holes: A technical note. Fatigue and Fracture of Engineering Materials and Structures, 27(9), pp. 879886, 2004. 